\title{
Mannitol-negative methicillin-resistant Staphylococcus aureus from nasal swab specimens in Brazil
}

\author{
Danielle Caldeira Martins dos Santos ${ }^{1}$, Thaina Miranda da Costa ${ }^{1}$, \\ Renata Fernandes Rabello ${ }^{2}$, Fábio Aguiar Alves ${ }^{1}$, Silvia Susana Bona de Mondino ${ }^{1}$ \\ ${ }^{1}$ Programa de Pós-gradução em Patologia, Faculdade de Medicina, \\ Universidade Federal Fluminense, Niterói, RJ, Brazil. \\ ${ }^{2}$ Departamento de Microbiologia e Parasitologia, Instituto Biomédico, \\ Universidade Federal Fluminense, Niterói, RJ, Brazil.
}

Submitted: June 4, 2013; Approved: August 15, 2014.

\begin{abstract}
The isolation of mannitol-negative methicillin-resistant Staphylococcus aureus from nasal swabs is reported. Among the 59 isolates, $9(15 \%)$ isolates were mannitol-negative; all of these isolates were categorized as staphylococcal cassette chromosome mec (SCCmec) type IVa. This report emphasizes that mannitol fermentation on mannitol salt agar should not be used as the sole criterion when screening nasal swab specimens for $S$. aureus.
\end{abstract}

Key words: mannitol-negative, MRSA, nasal swab surveillance, SCCmec.

Methicillin-resistant Staphylococcus aureus (MRSA) remains an important cause of nosocomial infections worldwide, with severe consequences for patients and significant healthcare costs. Active surveillance and correct identification of patients colonized with MRSA is an effective infection control strategy that helps prevent the spread of this organism within the hospital environment (Muto et al., 2003). Mannitol salt agar (MSA) is a conventional medium that is frequently used to screen nasal swab specimens for presumptive MRSA colonization based on the growth of yellow colonies derived from the fermentation of mannitol (Chapman, 1945). However, clinical isolates of mannitol-negative MRSA have been widely reported (Kateete et al., 2010; Shittu et al., 2007). The aim of this study was to describe the occurrence of $S$. aureus strains that do not ferment mannitol on MSA, which is an important screening method for the detection of MRSA colonization.

From August 2009 to August 2011 a total of 104 isolates presumed to be $S$. aureus were obtained via nasal swab surveillance of patients admitted to one public hospital and one private hospital located in the city of Niterói in Rio de Janeiro, Brazil. The strains were sub-cultured on MSA medium (Becton, Dickinson and Company, Sparks, Nevada, USA) and incubated at $35{ }^{\circ} \mathrm{C}$ for $24 \mathrm{~h}$ to confirm mannitol fermentation. Single colonies of Gram-positive, catalase-positive, mannitol-positive or-negative cocci were subjected to the tube coagulase and DNase tests. The tube coagulase test was performed using rabbit plasma (Laborclin, Curitiba, Paraná, Brazil). The DNase test was performed by incubating the isolates on DNase agar medium (Oxoid, Cambridge, UK). The isolates were tested for susceptibility to oxacillin $(1 \mu \mathrm{g})$ and cefoxitin $(30 \mu \mathrm{g})($ Cecon, São Paulo, São Paulo, Brazil) using the disk diffusion method, according to the guidelines of the Clinical and Laboratory Standards Institute (CLSI, 2012).

DNA extraction was performed as previously described by Pacheco et al. (1997). PCR amplification was performed using the primers 5'- GTG AAG ATA TAC CAA GTG ATT -3'and 5'- ATG CGC TAT AGA TTG AAA GGA T -3' (Eurofins, Huntsville, Alabama, USA), which are specific for the $m e c A$ gene, according to the methodology proposed by Zhang et al. (2005). PCR to detect the $n u c$ gene was performed according to the method described by Brakstad et al. (1992), using the primers 5'-GCG TTA ACG GAT GGT GAT GTT-3' and 5'-CAA AGC GCC ACG TTG TAA AAC AGC-3' (Eurofins, Huntsville, Alabama, USA). A multiplex-PCR assay was performed to characterize staphylococcal cassette chromo- 
Table 1 - General phenotypic characteristics of 104 Staphylococcus spp. according to nuc gene PCR results.

\begin{tabular}{|c|c|c|c|c|c|c|}
\hline \multirow[t]{2}{*}{ nис PCR } & \multicolumn{2}{|c|}{$\operatorname{MSA}^{*} \mathrm{n}(\%)$} & \multicolumn{2}{|c|}{ Coagulase n $(\%)$} & \multicolumn{2}{|c|}{ DNase n $(\%)$} \\
\hline & Positive & Negative & Positive & Negative & Positive & Negative \\
\hline Positive $(S$. aureus $) \mathrm{n}=69$ & $59(86)$ & $10(14)$ & $60(87)$ & $9(13)$ & $61(88)$ & $8(12)$ \\
\hline Negative (non-S. aureus) $\mathrm{n}=35$ & $31(89)$ & $4(11)$ & 0 & $35(100)$ & 0 & $35(100)$ \\
\hline
\end{tabular}

*MSA: mannitol salt agar.

some mec (SCCmec) types I to V in the MRSA strains according to the method developed by Zhang et al. (2005).

Of the 104 strains evaluated, 69 (66\%) strains were $S$. aureus, according to the results obtained after PCR amplification of the nuc gene, and 35 (34\%) strains were organisms other than $S$. aureus. The phenotypic characteristics of the strains are shown in Table 1.

Among the 69 S. aureus strains, 59 (86\%) strains harbored the mecA gene; of these MRSA strains, 15\% (9/59) were mannitol-negative but coagulase- and DNase-positive.

Of the 59 MRSA isolates examined, 43 (73\%) isolates were SCCmec-typeable with the primers used in this study. SCCmec type II was the most common (49\%) type, followed by type IV $(37 \%)$, including sub-types IVa $(n=14), \operatorname{IVb}(n=1)$, and IVc $(n=1)$; type III $(7 \%)$; type I $(5 \%)$ and type $\mathrm{V}(2 \%)$. The nine mannitol-negative strains were typed as SCCmec IVa.

The sensitivity and specificity of each test was calculated to evaluate the performance of the individual tests in the identification of $S$. aureus. The DNase test was the most sensitive test ( $88 \%$ sensitivity), followed by coagulase and MSA ( $87 \%$ and $86 \%$ sensitivity, respectively). The coagulase and DNase tests were the most specific tests $(100 \%$ specificity). The low specificity of mannitol (11\%) for detecting $S$. aureus could be caused by the large number of coagulase-negative staphylococci (CoNS) isolates that were mannitol-positive. Mannitol-negative $S$. aureus strains have also been reported by other authors (Kateete et al., 2010; Shittu et al., 2007). The identification of nine of these strains was correct based on the positivity of the coagulase and DNase tests. However, the isolation of MRSA strains with negative results for the coagulase (Bertrand et al., 2002; Del' Alamo et al., 2007) and DNAse (Rao et al., 2002) tests was described previously.

Comparing the presence of the mecA gene with the specificity of the disk diffusion method, 57 of the 59 MRSA isolates exhibited consistency levels of $100 \%$ $(57 / 57)$ and $75 \%(43 / 57)$ for cefoxitin and oxacilin, respectively. The cefoxitin disk test correlates well with $m e c A$ detection using PCR and also remains a good marker for the detection of methicillin resistance in $S$. aureus (Swenson et al., 2005). However, two of the 59 MRSA isolates were susceptible to both of the antimicrobials tested. This phenotype is known as pre-MRSA, in which the isolate harbors a repressed $m e c A$ gene; in this case, contact with $\beta$-lactam antibiotics could trigger the expression of the PBP2a protein (Berger-Bächi et al., 2002).

Worldwide, most HA-MRSA isolates are classified as types I, II and III; most CA-MRSA isolates belong to types IV and V (Chambers \& Deleo, 2009). The present study detected nasal MRSA isolates carrying SCCmec types I, II, III, IVa, IVb, IVc and V. The most common SCCmec detected was type II; this finding is a significant result that has been reported rarely. Silva-Carvalho et al. (2009) verified that SCCmec types III and IV were the most frequent among nasal MRSA isolates from two hospitals located in the Rio de Janeiro metropolitan region. Notably, all of the mannitol-negative MRSA isolates were categorized as type IVa.

In our study, we detected 35 CoNS isolates, of which $31(89 \%)$ isolates were mecA-positive. Most of these isolates were mannitol-positive $(28 / 31 ; 90 \%)$ but coagulaseand DNase test-negative. CoNS species that produce acid from mannitol have been described previously (Bannerman et al., 2007; Kateete et al., 2010). Carriage of the mecA gene is not exclusive to MRSA and has been reported frequently in several CoNS species isolated from human and animal sources (Mombach Pinheiro Machado et al., 2007; Black et al., 2009). This fact emphasizes the importance of CoNS as reservoirs of the mecA gene, which can be transmitted to $S$. aureus (Barbier et al., 2010; Tsubakishita et al., 2010).

According to our results, 9 (15\%) of the 59 MRSA strains identified in this study were mannitol-negative. Therefore, these strains would not have been detected using mannitol-positive colony growth on MSA as the sole criterion for MRSA screening. Thus, the inclusion of at least two additional tests, such as the coagulase tube and DNase tests, is needed to improve the identification of $S$. aureus strains and the early detection of patients who are infected or colonized by MRSA.

\section{References}

Bannerman TL, Peacock SJ (2007) Staphylococcus, Micrococcus and other catalase-positive cocci. In: Murray PR, Baron EJ, Jorgensen JH et al. (eds). Manual of Clinical Microbiology. ASM Press. Washington, pp 390-411.

Barbier F, Ruppé E, Hernandez D et al. (2010) Methicillinresistant coagulase-negative staphylococci in the community: high homology of SCCmec IVa between Staphylococcus epidermidis and major clones of methicillin-resistant Staphylococcus aureus. J Infect Dis 202:270-281. 
Berger-Bächi B, Rohrer S (2002) Factors influencing methicillin resistance in staphylococci. Arch Microbiol 178:165-171.

Bertrand X, Huguenin Y, Talon D (2002) First report of a catalase-negative methicillin-resistant Staphylococcus aureus. Diag Microbiol Infect Dis 43:245-246.

Black CC, Solyman SM, Eberlein LC et al. (2009) Identification of a predominant multilocus sequence type, pulsed-field gel electrophoresis cluster, and novel staphylococcal chromosomal cassette in clinical isolates of mecA-containing, methicillin-resistant Staphylococcus pseudintermedius. Vet Microbiol 139:333-338.

Brakstad OG, Aasbakk K, Maeland JA (1992) Detection of Staphylococcus aureus by Polymerase Chain Reaction Amplification of the nuc gene. J Clin Microbiol 30:1654-1660.

Chambers HF, DeLeo FR (2009) Waves of resistance: Staphylococcus aureus in the antibiotic era. Nat Rev Microbiol 7:629-641

Chapman GH (1945) The significance of sodium chloride in studies of staphylococci. J Bacteriol 50:201-203.

CLSI (2012) Performance standards for antimicrobial susceptibility testing. 22th Informational Supplement Update. Clinical and Laboratory Standards (M100-S22): Clinical and Laboratory Standards Institute. Wayne, PA.

Del'Alamo L, d'Azevedo PA, Strob AJ et al. (2007). Na outbreak of catalase-negative methicilin-resistant Staphylococcus aureus. J Hosp Infect 65:226-230.

Kateete DP, Kimani CN, Katabazi FA et al. (2010) Identification of Staphylococcus aureus: DNase and Mannitol salt agar improve the efficiency of the tube coagulase test. Annal Clin Microbiol Antimicrob 9:23-29.

Mombach Pinheiro Machado ABM, Reiter KC, Paiva RM, Barth AL (2007) Distribution of staphylococcal cassete chromosome mec (SCCmec) types I, II, III and coagulase negative staphylococci from patients attending a tertiary hospital in southern Brazil. J Med Microbiol 56:1328-1333.

Muto CA, Jernigan JA, Ostrowsky BE et al. (2003) SHEA Guideline for Preventing Nosocomial Transmission of Multidrug-Resistant Strains of Staphylococcus aureus and Enterococcus. Infect Control Hosp Epidemiol 24:362-386.
Pacheco AB, Guth BE, Soares KC et al. (1997) Random amplification of polymorphic DNA reveals serotype-specific clonal clusters among enterotoxigenic Escherichia coli strains isolated from humans. J Clin Microbiol 35:1521-1525.

Rao JG, Qamruddin AO, Hassan IA et al. (2002) Cluster of clinical isolates of epidemic methicillin-resistant Staphylococcus aureus (EMRSA) with a negative deoxyribonuclease (DNase) test - implications for laboratory diagnosis and infection control. J Hosp Infec 51:238-239.

Severin JA, Lestari ES, Kuntaman K et al. (2010) Nasal carriage of methicillin-resistant and methicillin-sensitive strains of Staphylococcus sciuru in the Indonesian population. Antimicron Agents Chemother 54:5413-5417.

Silva-Carvalho MC, Bonelli RR, Souza RR et al. (2009) Emergence of mul-tiresistant variants of the community-acquired methicillin-resistant Staphylococcus aureus lineage ST1SCCmecIV in two hospitals in Rio de Janeiro, Brazil. Diagn Microbiol Infect Dis 65:300-305.

Shittu A, Lin J, Morrison D (2007) Molecular identification and characterization of mannitol-negative methicillin-resistant Staphylococcus aureus. Diagn Microbiol Infect Dis 57:9395.

Swenson JM, Tenover FC and the Cefoxitin Disk Study Group (2005) Results of Disk diffusion testing with cefoxitin correlate with presence of mecA in Staphylococcus aureus. J Clin Microbiol 43:3818-3823.

Tsubakishita S, Kuwahara-Arai K, Sasaki T et al. (2010) Origin and molecular evolution of the determinant of methicillin resistance in staphylococci. Antimicrob Agents Chemother 54:4352-4359.

Zhang K, McClure, JA, Elsayed S et al. (2005) Novel multiplex PCR assay for characterization and concomitant subtyping of staphylococcal cassette chromosome mec types I to V in methicillin-resistant Staphylococcus aureus. J Clin Microbiol 43: 5026-5033.

Associate Editor: Elizabeth de Andrade Marques

All the content of the journal, except where otherwise noted, is licensed under a Creative Commons License CC BY-NC. 